\section{Amamentação e crescimento infantil: um estudo longitudinal em crianças do Rio de Janeiro, Brasil, 1999/2001}

\author{
Breastfeeding practices and infant growth: \\ a longitudinal study in Rio de Janeiro, \\ Brazil, 1999/2001
}

Maria Helena Constantino Spyrides 1,2 Cláudio José Struchiner 1,3 Maria Tereza Serrano Barbosa 4 Gilberto Kac ${ }^{5}$

\title{
Introdução
}

1 Programa de Computação Científica, Fundação Oswaldo Cruz,

Rio de Janeiro, Brasil.

2 Departamento de

Estatística, Universidade

Federal do Rio Grande

do Norte, Natal, Brasil.

3 Instituto de Medicina

Social, Universidade

do Estado do Rio de Janeiro,

Rio de Janeiro, Brasil.

4 Departamento de

Matemática e Estatística,

Universidade Federal

do Estado do Rio de Janeiro,

Rio de Janeiro, Brasil.

5 Instituto de Nutrição Josué

de Castro, Universidade

Federal do Rio de Janeiro,

Rio de Janeiro, Brasil.

Correspondência

M. H. C. Spyrides

Programa de Computação

Científica, Fundação

Oswaldo Cruz.

Av. Deputado Antônio

Florêncio Queiroz 2491,

Torre II, apto. 1901, Natal, RN

59092-500, Brasil.

mhspyrides@fiocruz.br

spyrides@ccet.ufrn.br

\section{Abstract}

Various studies have shown significant differences in growth patterns between breastfed and formula-fed infants. This paper aims to evaluate the effect of predominant breastfeeding $d u$ ration on anthropometric profile and to detect determinants associated with growth in Brazilian infants. Four hundred and seventy nine infants were studied in a health center in Rio de Janeiro through a longitudinal study with four follow-up waves at 0.5, 2, 6, and 9 months. The response variables were body weight and length, collected according to a standardized procedure. Data analysis was performed having longitudinal mixed-effects model as the main statistical procedure. Gestational age, birth weight, and birth length were positively associated with weight and length gains. The positive effect showed that the longer breastfeeding lasts, the greater the weight gains. Infants born through vaginal delivery presented lower weight gains than those born by cesarean delivery. The results emphasize the need for health programs encouraging breastfeeding practices up to the sixth month of life.

Breast Feeding; Nutritional Status; Child Welfare; Growth
A influência da amamentação no primeiro ano de vida é uma questão importante para avaliar o padrão de crescimento infantil. Estudos desenvolvidos em vários países vêm mostrando diferenças significativas no padrão de crescimento de crianças amamentadas ao seio e com fórmulas $1,2,3,4$. Há divergências quanto ao melhor momento de introduzir alimentos complementares 5,6 e até que ponto a amamentação ao seio supre as necessidades de nutrientes no primeiro ano de vida $7,8,9$.

$O$ efeito da amamentação sobre o crescimento infantil foi estudado por diversos autores, que constataram que as crianças amamentadas ao seio apresentavam inicialmente um maior crescimento em relação às amamentadas com fórmula, porém, a partir de um determinado momento, observava-se uma inversão nos ganhos de peso 4,10,11. Alguns autores questionam se essa redução pode ser atribuída ao fato de o leite materno não ser suficiente para alcançar as necessidades nutricionais para o crescimento das crianças após os quatro meses de idade 7 ou pela alimentação excessiva das amamentadas com fórmulas ou pela freqüência de mamadas ou pelo excesso de quantidade de leite oferecido 12. Por outro lado, há estudos que comprovam que crianças com maior duração da amamentação exclusiva podem acelerar o ganho de peso e de comprimento nos 
primeiros meses de vida, sem nenhum déficit aos 12 meses 13,14 ou mostrando crescimento na adolescência ainda maior do que as amamentadas com fórmulas precocemente 15.

Diante dessa controvérsia de resultados nos estudos, a questão permanece: as práticas alimentares levam a padrões diferenciados de crescimento infantil nos primeiros meses de vida? E que fatores podem estar associados ao crescimento infantil? As respostas a essas questões são importantes para esclarecer sobre a melhor conduta na orientação sobre a alimentação e cuidados mais adequados nessa fase de vida. Este artigo se propõe a identificar os determinantes do crescimento infantil nos primeiros meses de vida e avaliar o efeito da duração do aleitamento materno sobre a evolução do peso e comprimento das crianças acompanhadas durante os primeiros nove meses de vida, no Município do Rio de Janeiro, Brasil, utilizando modelos longitudinais lineares de efeitos mistos.

\section{Métodos}

\section{Captação e seleção dos participantes}

Os dados utilizados nesta análise fazem parte de uma pesquisa que avaliou a composição corporal e obesidade materna, acompanhando, simultaneamente, o crescimento infantil e informações sobre a amamentação. Os critérios de captação e seleção estão disponíveis em outras publicações 16,17,18. Trata-se de um estudo de coorte com nove meses de seguimento envolvendo mulheres brasileiras entre 15 e 45 anos, residentes no Município do Rio de Janeiro. O processo de coleta de dados durou 24 meses (15 de captação e 9 de seguimento) e foi desenvolvido no período de maio de 1999 a abril de 2001. Quatrocentas e setenta e nove mulheres foram recrutadas, e os recém-nascidos foram acompanhados segundo um desenho longitudinal com quatro medições aproximadamente aos 0,5; 2; 6 e 9 meses pós-parto.

\section{Critérios de elegibilidade e exclusão}

Os critérios de elegibilidade para ingresso na coorte foram: nascidos vivos de mães com idades entre 15 e 45 anos; recém-nascidos de mães com menos de trinta dias de pós-parto na data da primeira entrevista; a mãe não apresentar doenças crônicas; a mãe não ter dado à luz a gêmeos e morar na área programática do Centro Municipal de Saúde (CMS), situado no Município do Rio de Janeiro.

Como critério de exclusão, foram eliminadas as crianças cujos índices antropométricos estivessem fora dos limites biológicos estabelecidos pelas normas da Organização Mundial da Saúde (OMS) 19, que determina como aceitáveis os escores-z variando para os índices: peso/comprimento entre $-4 \mathrm{e}+5$; peso/idade entre -5 e +5 e comprimento/idade entre -5 e +3 .

\section{Variáveis estudadas}

O peso e comprimento das crianças foram obtidos em quatro momentos de visitas realizadas pela mãe e pela criança ao longo de nove meses pós-parto no CMS. Essas medidas antropométricas constituem as variáveis dependentes dos modelos em estudo.

As medidas de peso foram realizadas com a criança posicionada no colo da mãe, usando uma balança digital (Modelo PL 150, Filizola S.A., São Paulo, Brasil) com aproximação de $0,1 \mathrm{~kg}$. Primeiramente, a mãe era pesada e, em seguida, obtinha-se o peso da criança através da diferença de pesagens. O comprimento foi medido com aproximação a $0,1 \mathrm{~cm}$, com estadiômetro Kiddimetre (Child Growth Foundation, Reino Unido) com a criança deitada, seguindo as recomendações de Lohman et al. 20 .

A idade da criança foi calculada pela diferença entre a data de cada entrevista e a data de nascimento da mesma. Para efeito de melhor convergência do modelo, optou-se por trabalhar com a idade da criança expressa em meses. Outras variáveis relativas à criança consideradas como possíveis determinantes do crescimento infantil foram: sexo, idade gestacional (semanas), peso ao nascer $(\mathrm{kg})$ e comprimento ao nascer $(\mathrm{cm})$.

Para as informações sobre as categorias de aleitamento materno, utilizou-se a definição proposta pela Organização Pan-Americana da Saúde (OPAS) e pela OMS 21 que considera:

- Amamentação exclusiva: crianças que receberam somente leite materno, sem o uso de água, chá, suco ou papa;

- Amamentação predominante: crianças que receberam leite materno e outros líquidos, como água, chá ou sucos;

- Amamentação parcial: crianças que receberam leite materno e complementação com leite artificial e outros alimentos como água, chá, sucos e papas; 
- Alimentação artificial: crianças não recebendo mais leite materno.

Para compor a variável duração da amamentação, foram coletados os momentos em que a criança introduziu, em sua alimentação, novos itens, além do leite materno, de forma a obterse a duração para cada fase de alimentação especificada anteriormente. Considerou-se, para efeito de análise, a duração total (em meses) da amamentação predominante ao final do período estudado como a principal co-variável, já que um grupo muito pequeno de mães manteve amamentação exclusiva por mais tempo.

Além dessas, foram captadas informações referentes ao nível sócio-econômico da família, sobre o estado de saúde da mãe, hábitos pessoais e condições de vida. Essas informações foram obtidas através de entrevistas, nas quais foi preenchido um questionário em cada uma das quatro visitas da mãe ao CMS. Nesta análise, foram testadas as variáveis: tipo de parto (normal, cesáreo), estado civil da mãe (casada, união consensual, solteira), peso pré-gestacional da mãe $(\mathrm{kg})$, cor de pele da mãe (negra, parda, branca), hábito de fumar (fumante, nãofumante), anos de escolaridade ( $\leq 4,5-8,9-11$, $\geq 12$ anos), local de residência ao nascimento (urbana, rural), saneamento básico (rede de esgoto ou pluvial, fossa séptica, vala aberta) e renda familiar total $(<2 ; 2-4,9 ; \geq 5$ salários mínimos). O cálculo para conversão da renda em salários mínimos foi baseado nas informações do Departamento Intersindical de Estatística e Estudos Sócio-econômicos (DIEESE: http:// www.dieese.org.br/esp/salmin.html, acessado em 05/Nov/2003), valor de referência de abril de 2000, equivalente a $\mathrm{R} \$ 151,00$.

\section{Perdas de seguimento}

$\mathrm{Na}$ análise de dados longitudinais, é comum ocorrer perdas de seguimento. As taxas de seguimento foram calculadas pela relação entre o número de crianças que compareceram a cada uma das visitas subseqüentes e o número total de crianças no início do estudo. Essas taxas foram calculadas para as variáveis diretamente ligadas à criança, como: sexo (masculino, feminino), peso ao nascer (insuficiente: $<2.500 \mathrm{~g}$, baixo: $2.500-2.999 \mathrm{~g}$, normal: $\geq 3.000 \mathrm{~g}$ ), idade gestacional ( $<37$ semanas, $\geq 37$ semanas), tipo de parto (normal, cesáreo) e duração da amamentação $\left(<3\right.$, $\geq 3$ meses). O teste $\chi^{2}$ para proporções foi utilizado para avaliar se os padrões de perda de seguimento eram aleatórios ou indicavam seletividade.

\section{Modelagem estatística}

No estudo da evolução do crescimento infantil, medidas repetidas de uma mesma criança são observadas ao longo do tempo. Nesse caso, as observações de uma mesma criança tendem a ser correlacionadas entre si. As correlações entre as observações próximas no tempo são altas e, em geral, decrescem à medida que as distâncias entre estas aumentam. Na análise de medidas repetidas, é importante que os modelos incorporem possíveis dependências entre as observações tomadas de um mesmo indivíduo com o intuito de melhorar a precisão das estimativas.

As curvas de crescimento infantil são um exemplo disso, pois o interesse está centrado em avaliar a evolução das medidas antropométricas de crianças, como peso e comprimento, ao longo da idade. Sendo assim, é necessário ajustar um modelo que simultaneamente leve em consideração a estrutura média geral, como também sua variabilidade tanto entre quanto intra-indivíduos.

Os modelos de efeitos mistos permitem considerar o intercepto e/ou os coeficientes da regressão como efeitos aleatórios ou fixos. Esse modelo assume que o vetor de medidas repetidas de cada criança segue um modelo de regressão linear, no qual alguns dos parâmetros de regressão são específicos da população em estudo, ou seja, são os mesmos para todas as crianças, enquanto que outros parâmetros são específicos para cada uma das crianças. Isto é importante, pois nem sempre é possível o controle de todas as fontes de variação envolvidas no processo, como as decorrentes de fatores genéticos e biológicos, que são de difícil mensuração ou requerem um custo elevado para a obtenção da informação. Nesse aspecto, os modelos de efeitos mistos tornam-se bastante vantajosos, pois os efeitos aleatórios permitem controlar a variação existente entre crianças e que pode ser proveniente de fontes de variação não controladas no estudo.

Para a melhor compreensão sobre os parâmetros aleatórios, a Figura 1 mostra o comportamento de apenas seis das crianças envolvidas no estudo, refletindo a variação existente no padrão de crescimento entre as crianças. Pode-se observar que o comportamento do peso de cada uma das crianças poderia ser ajustado com interceptos e coeficientes de regressão diferentes, correspondendo ao peso inicial e à taxa de crescimento, respectivamente. Isto é, cada criança apresenta um ritmo de crescimento diferente do comportamento médio populacional (linha contínua), tanto no que se re- 


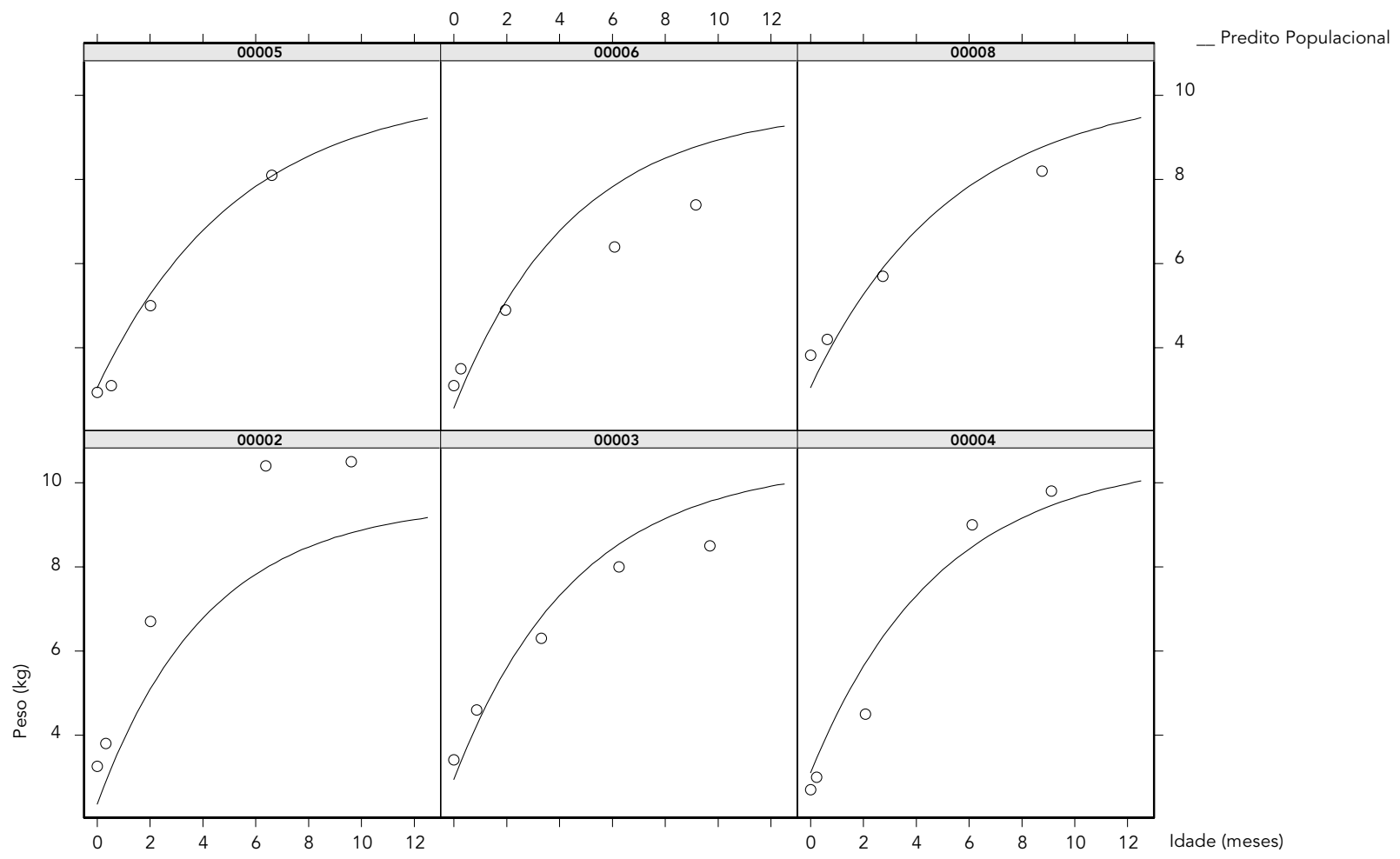

fere ao peso ao nascer, quanto nos ganhos de peso ao longo destes primeiros meses. O modelo de efeitos aleatórios, portanto, permite estimar a variação dos desvios individuais em torno da média populacional de cada parâmetro do modelo, fazendo com que as estimativas tornem-se mais precisas.

O modelo de efeitos mistos é dado pela equação 22,23:

$$
Y_{i}=X_{i} \beta+Z_{i} b_{i}+\varepsilon_{i}
$$

com efeitos fixos $\beta$ e efeitos específicos por indivíduo $b_{i}, \operatorname{com} b_{i} \sim N(0, D)$ e $\varepsilon_{i} \sim N\left(0, \sigma^{2} I\right)$. No modelo (1), $Y_{i}$ expressa o vetor resposta (peso ou comprimento) para a criança $i$, de dimensão $n_{i}$, com $1 \leq i \leq N, N$ correspondendo ao número de crianças. $X_{i}$ e $Z_{i}$ são matrizes de co-variáveis conhecidas.

Para cada uma das variáveis dependentes, peso e comprimento, procedeu-se inicialmente ao ajuste de um modelo reduzido, compreendendo as seguintes variáveis explicativas: duração da amamentação predominante (AMPRED), sexo e idade. Em seguida, examinou-se a im- portância da introdução de cada uma das variáveis explicativas e sua interação com as demais no modelo, a fim de considerar, para a construção do modelo final, apenas aquelas variáveis e interações que apresentaram significância inferior a $20,0 \%(\mathrm{p}<0,20)$, critério sugerido por Hosmer \& Lemeshow 24 .

Baseando-se nessas variáveis, cuja significância foi inferior a 0,20 , construiu-se os modelos finais separados para peso e comprimento com o auxílio do procedimento backward, para a seleção dos efeitos principais e, posteriormente, dos termos de interação. Para a escolha do melhor modelo, levou-se em consideração a comparação dos valores de AIC (Akaike Information Criterion) e do logaritmo da verossimilhança entre os modelos ajustados.

Várias estruturas da matriz de variância e co-variância foram testadas. Dois componentes estocásticos foram considerados na modelagem: o dos efeitos aleatórios e o da correlação serial entre as medidas repetidas. Para modelar a estrutura de variância dos efeitos alea- 
tórios, ajustou-se uma matriz não estruturada, e a que melhor descreveu as correlações seriais entre as medidas repetidas de uma mesma criança, nesse caso, foi a matriz de correlação exponencial espacial, que leva em consideração as distâncias no tempo entre observações.

\section{Resultados}

A população alvo constituiu-se por famílias de baixa renda, portanto, os fatores econômicos apresentaram pouca variabilidade, não tendo impacto significativo sobre a variável resposta. As informações sobre as condições de moradia revelaram que quase todos os domicílios eram servidos com água encanada dentro de casa, com sanitários ou banheiros e o principal sistema de escoamento sendo a rede de esgoto ou pluvial.

As mães entrevistadas tiveram seu primeiro parto com idade média de 20,7 anos, sendo que $45,2 \%$ são primíparas. Para as demais mulheres, o intervalo médio interpartal foi de $59 \mathrm{me}$ ses. O parto normal foi o mais praticado $(62,2 \%)$, seguido da cesariana $(37,8 \%)$.

O tempo médio de escolaridade foi de sete anos, sendo que mais da metade das mães entrevistadas cursaram somente o ensino fundamental. A renda mensal média familiar foi de 4,32 salários mínimos, sendo que mais de 70,0\% dessas famílias têm renda inferior a cinco salários mínimos (Tabela 1 ).

A população em estudo constituiu-se de 236 meninos e 243 meninas, totalizando 479 crianças acompanhadas durante os nove primeiros meses de vida. O peso médio ao nascer foi de $3.254,3 \mathrm{~g} \pm 507,2$ e $3.115,0 \mathrm{~g} \pm 478,4$ para meninos e meninas, respectivamente, e o comprimento médio ao nascer foi de $49,3 \mathrm{~cm} \pm 2,4$ para os meninos e $48,6 \mathrm{~cm} \pm 2,4$ para as meninas. Os bebês nasceram com idade gestacional média de 38,6 semanas, variando de 30 a 42 semanas. O número de partos prematuros $(<37$ semanas) ocorridos entre essas crianças correspondeu a $11,3 \%$ entre meninos e $9,0 \%$ entre as meninas.

$\mathrm{Na}$ primeira entrevista, $11,5 \%$ das mães complementavam o leite de peito com outro tipo de leite. Ao final do estudo, 40,0\% das crianças estavam tomando outro leite sem ser de peito, e 40,0\% misturavam leite de peito com outro tipo. A duração mediana de amamentação predominante foi de 67 dias, aproximadamente dois meses, sendo que $27,0 \%$ amamentaram predominantemente por um mês ou menos (Tabela 1). Os prematuros apresentaram uma duração mediana de amamentação pre-
Tabela 1

Características selecionadas de mães e filhos Rio de Janeiro, Brasil, 1999/2001.

\begin{tabular}{|c|c|}
\hline & $\%$ \\
\hline \multicolumn{2}{|l|}{ Variáveis da criança } \\
\hline \multicolumn{2}{|l|}{ Peso ao nascer $(\mathrm{kg})$} \\
\hline Insuficiente: $<2,5$ & 8,2 \\
\hline Baixo: 2,5-2,9 & 24,3 \\
\hline Normal: $\geq 3,0$ & 67,5 \\
\hline \multicolumn{2}{|c|}{ Introdução de papas (meses) } \\
\hline$<4$ & 11,2 \\
\hline $4-4,9$ & 33,0 \\
\hline $5-5,9$ & 33,0 \\
\hline$\geq 6$ & 22,8 \\
\hline \multicolumn{2}{|c|}{$\begin{array}{l}\text { Duração da amamentação } \\
\text { predominante (meses) }\end{array}$} \\
\hline$<1$ & 27,0 \\
\hline $1-2,9$ & 29,7 \\
\hline $3-5,9$ & 32,7 \\
\hline$\geq 6$ & 10,7 \\
\hline \multicolumn{2}{|l|}{ Variáveis da mãe } \\
\hline \multicolumn{2}{|c|}{ Renda mensal (salários mínimos) } \\
\hline$<2$ & 32,4 \\
\hline $2-4,9$ & 40,8 \\
\hline$\geq 5$ & 26,8 \\
\hline \multicolumn{2}{|c|}{ Nível de escolaridade (anos) } \\
\hline $1-4$ & 30,0 \\
\hline $5-8$ & 42,4 \\
\hline $9-11$ & 25,2 \\
\hline$\geq 12$ & 2,3 \\
\hline \multicolumn{2}{|l|}{ Tipo de parto } \\
\hline Normal & 62,2 \\
\hline Cesáreo & 37,8 \\
\hline
\end{tabular}

dominante de 21 dias, enquanto que as nascidas a termo, de 90 dias.

Neste estudo, das 479 crianças acompanhadas, constatou-se que 61 só haviam comparecido a uma entrevista, 88 , a duas, 90 , a três, e 238 crianças completaram as quatro visitas ao longo dos nove meses. Os padrões de perdas de seguimento foram considerados aleatórios para todas as co-variáveis incluídas no modelo (Tabela 2).

Comparou-se o valor do AIC para várias estruturas da matriz de variância e co-variância. Para a variável peso, a matriz não estruturada para os efeitos aleatórios e a do tipo exponencial espacial para a correlação serial entre me- 
didas repetidas foram as que apresentaram o menor valor para AIC para o modelo $(1753,9)$. Para o comprimento, a matriz diagonal e a exponencial espacial apresentaram melhor ajuste $(4540,5)$. Os valores do AIC do modelo completo comparado ao AIC do modelo reduzido para o peso $(2603,3)$ e comprimento $(5631,0)$ revelaram a importância da introdução das variáveis explicativas nos modelos.

A Tabela 3 indica a existência de interação entre sexo e idade, o que mostra um crescimento diferencial entre os sexos de acordo com a idade (valor-p $=0,0010$ ). O coeficiente positivo mostrou que quanto maior a duração da amamentação predominante, maior é o peso infantil.

O peso ao nascer e a idade gestacional mostraram um coeficiente positivo indicando que a criança prematura e a que nasce com baixo peso têm uma evolução do peso menor que as demais. Uma constatação interessante no estudo foi que crianças nascidas de parto normal apresentam pesos menores que as nascidas através de cesariana. Realizou-se um teste quiquadrado para proporções para verificar a possibilidade de uma possível associação entre o tipo de parto e cada uma das variáveis: idade gestacional e peso ao nascer. Nenhum dos dois testes confirmou associação do tipo de parto com a idade gestacional (valor- $p=0,7158$ ) ou com o peso ao nascer (valor-p $=0,3158$ ). Isto é, não há nenhuma tendência de crianças nascidas com parto cesáreo serem prematuras ou de baixo peso ao nascer.

Não se constatou efeito significativo do padrão de amamentação sobre o comprimento infantil (Tabela 4). Verificou-se que o comprimento e o peso ao nascer e a interação sexo por idade são fortes preditores da evolução do comprimento no primeiro ano de vida.

\section{Discussão}

Os resultados desta investigação apontam para a influência da duração da amamentação predominante, idade gestacional, peso ao nascer e tipo de parto como determinantes da evolução do peso nos primeiros meses de vida. Nem a duração da amamentação predominante, nem o tipo de parto, no entanto, interferem no comprimento infantil nos primeiros meses de vida.

Este estudo mostra a influência da duração da amamentação predominante sobre a evolução do peso, mas não sobre o comprimento nos primeiros meses de vida. Quanto maior a duração da amamentação predominante maior o peso da criança pelo menos até os nove primei-
Tabela 2

$$
\begin{aligned}
& \text { Taxa de seguimento completo por momento da entrevista. } \\
& \text { Rio de Janeiro, Brasil, 1999/2001. }
\end{aligned}
$$

\begin{tabular}{|c|c|c|c|c|}
\hline \multirow[t]{3}{*}{ Variáveis } & \multicolumn{4}{|c|}{ Meses } \\
\hline & 0,5 & 2 & 6 & 9 \\
\hline & $\mathrm{n}$ & \multicolumn{3}{|c|}{ Taxa de seguimento (\%) } \\
\hline \multicolumn{5}{|l|}{ Sexo } \\
\hline Masculino & 236 & 87,8 & 65,2 & 57,4 \\
\hline \multirow[t]{2}{*}{ Feminino } & 242 & 85,6 & 69,2 & 59,5 \\
\hline & & $(0,4892)^{*}$ & $(0,3595)$ & $(0,6449)$ \\
\hline \multicolumn{5}{|l|}{ Peso ao nascer $(\mathrm{kg})$} \\
\hline Insuficiente: $<2,5$ & 39 & 82,0 & 71,8 & 53,8 \\
\hline Baixo: 2,5-2,9 & 116 & 90,1 & 73,0 & 61,3 \\
\hline \multirow[t]{2}{*}{ Normal: $\geq 3,0$} & 323 & 86,1 & 64,7 & 58,0 \\
\hline & & $(0,3805)$ & $(0,2260)$ & $(0,6966)$ \\
\hline \multicolumn{5}{|c|}{ Idade gestacional (semanas) } \\
\hline$<37$ & 74 & 98,6 & 78,4 & 67,6 \\
\hline \multirow[t]{2}{*}{$\geq 37$} & 315 & 98,0 & 76,2 & 65,7 \\
\hline & & $(0,7473)$ & $(0,6891)$ & $(0,7619)$ \\
\hline \multicolumn{5}{|l|}{ Tipo de parto } \\
\hline Normal & 219 & 98,1 & 92,1 & 78,0 \\
\hline \multirow[t]{2}{*}{ Cesáreo } & 133 & 98,4 & 90,7 & 81,4 \\
\hline & & $(0,8273)$ & $(0,6614)$ & $(0,4571)$ \\
\hline \multicolumn{5}{|c|}{$\begin{array}{l}\text { Duração da amamentação } \\
\text { predominante (meses) }\end{array}$} \\
\hline$<3$ & 133 & 96,2 & 95,5 & 82,7 \\
\hline \multirow[t]{2}{*}{$\geq 3$} & 195 & 99,0 & 93,8 & 81,5 \\
\hline & & $(0,0992)$ & $(0,5213)$ & $(0,7868)$ \\
\hline
\end{tabular}

* Valor-p do teste $\chi^{2}$ para proporções.

ros meses. Portanto, este estudo confirma que as práticas alimentares levam a padrões diferenciados de crescimento infantil no que se refere ao peso infantil, devendo ser mantidas as recomendações da OMS. Victora et al. 25 constataram que não existe diferença significativa do crescimento entre o grupo de crianças amamentadas exclusiva e predominantemente. Dado que o número de mães que mantêm a amamentação exclusiva é muito pequeno, poderse-ia sugerir que, pelo menos, a amamentação predominante se mantivesse até os seis meses de vida.

Além disso, não se pode esquecer que o aleitamento materno até os seis meses de vida é importante não só para o crescimento infantil, como também por seus benefícios comprovados na proteção contra doenças infecciosas e respiratórias $13,26,27,28$. O melhor momento para o desmame ou introdução de alimentos complementares deve levar em conta esses benefícios que contribuem para a redução da morbi-mortalidade infantil. No decorrer do pri- 
Modelo linear de efeitos mistos para a evolução do peso $(\mathrm{kg})$ de crianças menores de 1 ano Rio de Janeiro, Brasil, 1999/2001.

\begin{tabular}{|c|c|c|c|}
\hline Variáveis & Estimativa & Erro padrão & Valor-p \\
\hline \multicolumn{4}{|l|}{ Efeitos fixos } \\
\hline Intercepto & $-2,4600$ & 0,5727 & $<0,0001$ \\
\hline Idade (meses) & 1,1719 & 0,0151 & $<0,0001$ \\
\hline Efeito quadrático (meses) & $-0,0581$ & 0,0014 & $<0,0001$ \\
\hline \multicolumn{4}{|l|}{ Sexo } \\
\hline Masculino & & & - \\
\hline Feminino & $-0,0323$ & 0,0193 & 0,0964 \\
\hline Duração da amamentação predominante (meses) & 0,0379 & 0,0092 & $<0,0001$ \\
\hline Peso ao nascer $(\mathrm{kg})$ & 0,7203 & 0,0588 & $<0,0001$ \\
\hline Idade gestacional (semanas) & 0,0451 & 0,0118 & 0,0002 \\
\hline \multicolumn{4}{|l|}{ Tipo de parto } \\
\hline Normal & - & - & - \\
\hline Cesáreo & 0,0407 & 0,0193 & 0,0356 \\
\hline Comprimento ao nascer $(\mathrm{cm})$ & 0,0297 & 0,0118 & 0,0127 \\
\hline \multicolumn{4}{|l|}{ Efeito de interação } \\
\hline \multicolumn{4}{|l|}{ Sexo $x$ idade } \\
\hline Masculino & - & - & - \\
\hline Feminino & $-0,0250$ & 0,0076 & 0,0010 \\
\hline \multirow[t]{2}{*}{ Efeitos aleatórios } & \multicolumn{3}{|c|}{ Intervalos de confiança $95 \%$} \\
\hline & Limite inferior & Estimativa & Limite superior \\
\hline$\sigma_{\text {intercepto }}$ & 0,0266 & 0,0551 & 0,1140 \\
\hline$\sigma_{\text {idade }}$ & 0,1066 & 0,1180 & 0,1307 \\
\hline$\sigma_{\text {int, idade }}$ & $-1,0000$ & 0,9978 & 1,0000 \\
\hline$\rho$ & 0,6471 & 1,1032 & 1,8808 \\
\hline$\sigma^{2}$ residual & 0,1118 & 0,1260 & 0,1420 \\
\hline -2 Log-verossimilhança & 1723,9 & & \\
\hline $\mathrm{AIC}$ & 1753,9 & & \\
\hline
\end{tabular}

meiro ano, deve-se ter uma boa orientação dos tipos de alimentos que podem ser introduzidos na alimentação infantil, de forma a atender as necessidades de nutrientes fundamentais para o crescimento e desenvolvimento infantis. $\mathrm{O}$ tipo de alimentação no decorrer desse primeiro ano deve respeitar o amadurecimento do sistema gastrintestinal e da capacidade que a criança tem de metabolizar seus componentes 29 .

O prematuro ao nascer, independentemente das características econômicas e sociais da família, implica um risco maior de desenvolver desnutrição na infância, com todas as desvantagens que isso representa para o desenvolvimento do indivíduo. Os resultados deste estudo apontam para a importância dos cuidados gestacionais e da saúde da mãe sobre o crescimento da criança nos primeiros meses de vida, tendo em vista a significância do baixo peso ao nascer e da idade gestacional como determinantes do crescimento infantil nos primeiros meses de vida. A evolução do peso nessa fase de idade em crianças prematuras tende a ser mais baixa que em crianças nascidas a termo. Os resultados apresentados mostram que quanto maior o peso da criança ao nascer, maior será o peso no primeiro ano de vida.

Um dos fatores importantes na evolução do peso infantil ao longo dos primeiros meses encontrado neste estudo foi o tipo de parto. A análise mostrou que crianças nascidas de parto normal tendem a pesar menos do que crianças nascidas de parto cesáreo. Poder-se-ia supor que crianças nascidas através de cesariana teriam alguma tendência a nascer prematura, porém os testes não confirmaram essa associa- 
Modelo linear de efeitos mistos para a evolução do comprimento $(\mathrm{cm})$ de crianças menores de 1 ano.

Rio de Janeiro, Brasil, 1999/2001.

\begin{tabular}{|c|c|c|c|}
\hline Variáveis & Estimativa & Erro padrão & Valor-p \\
\hline \multicolumn{4}{|l|}{ Efeitos fixos } \\
\hline Intercepto & 20,7264 & 1,8662 & $<0,0001$ \\
\hline Idade (meses) & 4,0169 & 0,0392 & $<0,0001$ \\
\hline Efeito quadrático (meses) & $-0,1746$ & 0,0039 & $<0,0001$ \\
\hline \multicolumn{4}{|l|}{ Sexo } \\
\hline Masculino & - & - & \\
\hline Feminino & $-0,1863$ & 0,0689 & 0,0072 \\
\hline Peso ao nascer $(\mathrm{kg})$ & 1,7506 & 0,2009 & $<0,0001$ \\
\hline Idade gestacional (semanas) & 0,2006 & 0,0381 & $<0,0001$ \\
\hline Comprimento ao nascer $(\mathrm{cm})$ & 0,2807 & 0,0395 & $<0,0001$ \\
\hline \multicolumn{4}{|l|}{ Efeito de interação } \\
\hline \multicolumn{4}{|l|}{ Sexo $x$ idade } \\
\hline Masculino & - & - & \\
\hline Feminino & $-0,0639$ & 0,0149 & $<0,0001$ \\
\hline \multirow[t]{2}{*}{ Efeitos aleatórios } & \multicolumn{3}{|c|}{ Intervalos de confiança 95\% } \\
\hline & Limite inferior & Estimativa & Limite superior \\
\hline$\sigma_{\text {intercepto }}$ & 0,8215 & 0,9305 & 1,0540 \\
\hline$\sigma_{\text {idade }}$ & 0,1780 & 0,2017 & 0,2284 \\
\hline$\rho$ & 0,7049 & 0,9303 & 1,2277 \\
\hline$\sigma^{2}$ residual & 0,9126 & 1,0303 & 1,1631 \\
\hline -2 Log-verossimilhança & 4516,5 & & \\
\hline AIC & 4540,5 & & \\
\hline
\end{tabular}

ção. Por outro lado, a cesariana tende a ser feita quando as crianças são grandes ao nascer, ou seja, com maior peso e comprimento ao nascer 30 , o que poderia causar um confundimento no estudo. No entanto, não se constatou evidência de diferença significativa do peso ou comprimento ao nascer entre os tipos de parto realizados nessa comunidade.

Perez-Escamilla et al. 31 estudaram o impacto da cesariana sobre o início e duração da amamentação em uma pesquisa desenvolvida no México, em 1987. Os autores constataram que a cesariana é um fator de risco para não iniciar a amamentação e para a duração da amamentação por menos de um mês. Chapman \& Perez-Escamilla 32 verificaram que as mães que iniciam a amamentação antes de 72 horas pósparto tendem a prolongar o tempo de amamentação ao seio. Rajan 33 constatou que o tipo do parto, método de indução do trabalho de parto, técnicas de relaxamento e respiração, peso da criança ao nascer e a saúde física da mãe estão associados com a amamentação nas pri- meiras seis semanas pós-parto. Portanto, a redução no desconforto pós-cirúrgico do parto cesáreo pode contribuir para a melhoria na duração da amamentação. No entanto, outros autores não identificaram associação entre a duração da amamentação e o tipo de parto e nem com o tempo inicial do aleitamento materno 34 .

Por outro lado, o estudo de Patel et al. 35 mostrou que o maior tempo de internação depois de uma cesariana pode ajudar as mães a iniciar a amamentação, tornando-as mais propensas a alcançarem a amamentação exclusiva no momento da alta hospitalar. Embora vários artigos que discutem a relação entre tipo de parto e duração da amamentação estejam disponíveis na literatura, nenhum aborda a influência do parto sobre a evolução do peso da criança nos primeiros meses de vida.

A modelagem utilizada neste estudo constituiu-se de modelos lineares de efeitos mistos que permitem, em um primeiro estágio, considerar que as unidades amostrais sejam selecionadas aleatoriamente da população de interes- 
se e, em um segundo estágio, que um número de medidas sejam observadas de cada unidade do primeiro estágio. Esta abordagem tem a flexibilidade de especificar alguns efeitos como fixos e outros aleatórios, levando em consideração a estrutura de variância existente intra e entre indivíduos. Dessa forma, fornecem estimativas mais precisas, o que torna o teste mais sensível para captar diferenças significativas em relação aos estudos transversais. Ainda são raros os estudos que utilizam essa modelagem para testar o efeito da amamentação sobre o crescimento infantil 36 .

A análise de dados longitudinais geralmente apresenta algumas limitações como problemas de dados faltantes ou incompletos em decorrência da perda de seguimento que podem causar problemas de viés na amostra investigada. No entanto, constatou-se que as perdas de seguimento foram aleatórias segundo as variáveis de interesse no modelo.
Este estudo reforça a necessidade de programas que promovam o acompanhamento das mães durante a gravidez de forma a evitar as complicações que possam resultar na prematuridade e no baixo peso ao nascer. A orientação sobre a dieta da mãe, o controle do seu estado nutricional e outros cuidados importantes durante a gravidez podem evitar complicações para a saúde do bebê, favorecendo o peso ao nascer e a idade gestacional adequados ao crescimento futuro da criança. Além disso, os programas que encorajem o aleitamento materno predominante até os seis meses de vida devem ser continuados. A alimentação complementar deve seguir orientação rigorosa de profissionais que avaliem as necessidades nutricionais individuais para um crescimento e um desenvolvimento infantil saudáveis.

\section{Resumo}

Vários estudos vêm demonstrando diferenças significativas no padrão de crescimento entre crianças amamentadas ao seio e com fórmulas. O objetivo deste estudo é avaliar o efeito da duração da amamentação predominante sobre o perfil antropométrico e identificar os determinantes do crescimento infantil em uma coorte de crianças brasileiras. Foram acompanhadas 479 crianças em um centro de saúde do Rio de Janeiro, através de um estudo longitudinal envolvendo quatro seguimentos: 0,5; 2; 6 e 9 meses. As variáveis dependentes foram o peso e o comprimento, coletados de acordo com procedimentos padronizados. A análise longitudinal foi desenvolvida através do modelo linear de efeitos mistos. A idade gestacional, o peso e o comprimento ao nascer mostraram associação significativa com a evolução de peso e de comprimento. Constatou-se que quanto maior a duração da amamentação predominante, maior o peso infantil. As crianças nascidas por parto cesáreo apresentaram pesos mais elevados do que aquelas nascidas por parto normal. Estes resultados reforçam a necessidade de programas que encorajem o aleitamento materno até os seis meses.

Aleitamento Materno; Estado Nutricional; Bem-estar da Criança; Crescimento 


\section{Colaboradores}

M. H. C. Spyrides participou na análise estatística e na redação do manuscrito. C. J. Struchiner participou em várias fases do estudo, incluindo interpretação dos dados e redação do manuscrito. M. T. S. Barbosa participou na interpretação dos dados e redação do manuscrito. G. Kac participou em todas as fases do estudo, incluindo concepção, desenho, seguimento, interpretação dos dados e redação do manuscrito.

\section{Agradecimentos}

Conselho Nacional de Desenvolvimento Científico e Tecnológico (CNPq), Coordenação de Aperfeiçoamento de Pessoal de Nível Superior (CAPES), Fundação Universitária José Bonifácio da Universidade Federal do Rio de Janeiro (FUJB/UFRJ) e Fundação Carlos Chagas Filho de Amparo à Pesquisa do Estado do Rio de Janeiro (FAPERJ).

\section{Referências}

1. Dewey KG, Peerson JM, Brown KH, Krebs NF, Michaelsen KF, Persson LA, et al. Growth of breastfed infants deviates from current reference data: a pooled analysis of US, Canadian, and European data sets. Pediatrics 1995; 96 (3 Pt 1):495-503.

2. Dewey KG, Cohen RJ, Brown KH, Rivera LL. Effects of exclusive breastfeeding for four versus six months on maternal nutritional status and infant motor development: results of two randomized trials in Honduras. J Nutr 2001; 131:262-7.

3. Haschke F, van't Hof MA. Euro-growth references for BF boys and girls: influence of breast-feeding and solids on growth until 36 months of age. J Pediatr Gastroenterol Nutr 2000; 31 Suppl 1:S60-71.

4. De Onis M, Onyango AW. The Centers for Disease Control and Prevention 2000 growth charts and the growth of breastfed infants. Acta Paediatr 2003; 92:413-9.

5. Hop LT, Gross R, Giay T, Sastroamidjojo S, Schultink W, Lang NT. Premature complementary feeding is associated with poorer growth of Vietnamese children. J Nutr 2000; 130:2683-90.

6. Fewtrell MS, Lucas A, Morgan JB. Factors associated with weaning in full term and preterm infants. Arch Dis Child Fetal Neonatal Ed 2003; 88:296-301.

7. Naing KM, Co TT. Growth and milk intake of exclusively breast-fed Myanmar infants. Eur J Clin Nutr 1991; 45:203-7.

8. Diaz S, Herreros C, Aravena R, Casado ME, Reyes MV, Schaippacasse V. Breast-feeding duration and growth of fully breast-fed infants in a poor urban Chilean population. Am J Clin Nutr 1995; 62:371-6.

9. Abiona TC, Onayade AA, Ijadunola KT, Abayomi IO, Makanjuola RO. Growth pattern of exclusively breast-fed infants during the first six months of life in Ile-Ife, Osun State, Nigeria. Nutr Health 2002; 16:301-12.

10. Dewey KG. Growth characteristics of BF compared to formula-fed infants. Biol Neonate 1998; 74:94105.

11. Donma MM, Donma O. Infant feeding and growth: a study on Turkish infants from birth to 6 months. Pediatr Int 1999; 41:542-8.

12. Yoneyama K, Nagata $H$, Asano $H$. Growth of Japonese breast-fed and bottle-fed infants from birth to 20 months. Ann Hum Biol 1994; 21:597608 .

13. Kramer MS, Guo T, Platt RW, Shapiro S, Collet JP, Chalmers B, et al. Breastfeeding and infant growth: biology or bias? Pediatrics 2002; 110 (2 Pt 1):343-7.

14. Kramer MS, Guo T, Platt RW, Sevkovskaya Z, Dzikovich I, Collet JP, et al. Infant growth and health outcomes associated with 3 compared with 6 mo of exclusive breastfeeding. Am J Clin Nutr 2003; 78:291-5.

15. Martin RM, Smith GD, Mangtani P, Frankel S, Gunnell D. Association between breast feeding and growth: the Boyd-Orr cohort study. Arch Dis Child Fetal Neonatal Ed 2002; 87:F193-201.

16. Kac G, Benício MHDA, Valente JG, VelásquezMeléndez G. Postpartum weight retention among women in Rio de Janeiro: a follow-up study. Cad Saúde Pública 2003; 19 Suppl 1:S149-61. 
17. Kac G, Benício MHDA, Velásquez-Meléndez G, Valente JG, Struchiner CJ. Breastfeeding and postpartum weight retention in a cohort of Brazilian women. Am J Clin Nutr 2004; 79:487-93.

18. Kac G, Benício MHDA, Velásquez-Meléndez G, Valente JG, Struchiner CJ. Gestational weight gain and prepregnancy weight influence postpartum weight retention in a cohort of Brazilian women. J Nutr 2004; 134:661-6.

19. World Health Organization. Physical status: the use and interpretation of anthropometry. Geneva: World Health Organization; 1995. (WHO Technical Report Series 854).

20. Lohman TG, Roche AF, Martorell R. Anthropometric standardization reference manual. Champaign: Human Kinetics Books; 1988.

21. Organización Panamericana de la Salud/Organización Mundial de la Salud. Indicadores para evaluar las prácticas de lactancia Materna. Washington DC: Centro de Estudio y Documentación, Organización Mundial de la Salud; 1991.

22. Pinheiro JC, Bates DM. Mixed-effects models in S and S-PLUS. New York: Springer-Verlag; 2000.

23. Verbeke G, Molenberghs G. Linear mixed for longitudinal data. New York: Springer-Verlag; 2000

24. Hosmer DW, Lemeshow S. Applied logistic regression. New York: Wiley; 1989.

25. Victora CG, Morris SS, Barros FC, Horta BL, Weiderpass E, Tomasi E. Breast-feeding and growth in Brazilian infants. Am J Clin Nutr 1998; 67:452-8.

26. Huffman SL, Combest C. Role of breast-feeding in the prevention and treatment of diarrhea. J Diarrhoeal Dis Res 1990; 8:68-81.

27. Dewey KG, Heinig MJ, Nommsen-Rivers LA. Differences in morbidity between breast-fed and formula-fed infants. J Pediatr 1995; 126 (5 Pt 1):696702.
28. Villalpando S, Lopez-Alarcon M. Growth faltering is prevented by breast-feeding in underprivileged infants from Mexico City. J Nutr 2000; 130:546-52.

29. Bronner YL, Paige DM. Current concepts in infant nutrition. J Nurse Midwifery 1992; 37 (2 Suppl): 43S-58S.

30. Fortney JA, Higgins JE, Kennedy KI, Laufe LE, Wilkens L. Delivery type and neonatal mortality among 10,749 breeches. Am J Public Health 1986; 76:980-5.

31. Perez-Escamilla R, Maulen-Radovan I, Dewey KG. The association between cesarean delivery and breast-fedding outcomes among Mexican women. Am J Public Health 1996; 86:832-6.

32. Chapman DJ, Perez-Escamilla R. Does delayed perception of the onset of lactation shorten breastfeeding duration? J Hum Lact 1999; 15:107-11.

33. Rajan L. The impact of obstetric procedures and analgesia/anaesthesia during labour and delivery on breastfeeding. Midwifery 1994; 10:87-103.

34. Kearney MH, Cronenwett LR, Reinhardt R. Cesarean delivery and breastfeeding outcomes. Birth 1990; 17:97-103.

35. Patel RR, Liebling RE, Murphy DJ. Effect of operative delivery in the second stage of labor on breastfeeding success. Birth 2003; 330:255-60.

36. Baxter-Jones ADG, Cardy AH, Helms PJ, Phillips DO, Smith WCS. Influence of socioeconomic conditions on growth in infancy: the 1921 Aberdeen birth cohort. Arch Dis Child 1999; 81:5-9.

Recebido em 12/Ago/2004

Versão final reapresentada em 09/Nov/2004 Aprovado em 23/Nov/2004 